Cite this: Phys. Chem. Chem. Phys., 2011, 13, 20779-20786

\title{
Spectroscopic study of the electric field induced valence change of Fe-defect centers in $\mathrm{SrTiO}_{3}$
}

\author{
Christian Lenser, ${ }^{* a b}$ Aleksandr Kalinko,${ }^{c}$ Alexei Kuzmin, ${ }^{c}$ Dzintars Berzins, ${ }^{c}$ \\ Juris Purans, ${ }^{c}$ Kristof Szot, ${ }^{a d}$ Rainer Waser $^{a e}$ and Regina Dittmann ${ }^{a b}$
}

Received 16th June 2011, Accepted 27th September 2011

DOI: $10.1039 / \mathrm{c1cp21973a}$

The electrochemical changes induced by an electric field in Fe-doped $\mathrm{SrTiO}_{3}$ have been investigated by X-ray absorption spectroscopy (XANES and EXAFS), electron paramagnetic resonance (EPR) and Raman spectroscopy. A detailed study of the Fe dopant in the regions around the anode and cathode reveals new insights into the local structure and valence state of $\mathrm{Fe}$ in $\mathrm{SrTiO}_{3}$ single crystals. The ab initio full multiple-scattering XANES calculations give an evidence of the oxygen vacancy presence in the first coordination shell of iron. Differences in the length and disorder of the $\mathrm{Fe}-\mathrm{O}$ bonds as extracted from EXAFS are correlated to the unequivocal identification of the defect type by complementary spectroscopical techniques to identify the valence state of the $\mathrm{Fe}$-dopant and the presence of the $\mathrm{Fe}-\mathrm{V}_{\ddot{O}}$ complexes. Through this combinatorial approach, novel structural information on $\mathrm{Fe}-\mathrm{V}_{\ddot{O}}$ complexes is provided by $\mathrm{X}$-ray absorption spectroscopy, and the relation of $\mathrm{Fe}-\mathrm{O}$ bond length, doping level and oxidation state in $\mathrm{SrTi}_{1-x} \mathrm{Fe}_{x} \mathrm{O}_{3}$ is briefly discussed.

\section{Introduction}

In recent years, considerable effort has been spent on the investigation of the valence state of $3 d$ transition metal dopants in the perovskite $\mathrm{SrTiO}_{3}$ by the use of X-ray absorption spectroscopy. In Fe-doped $\mathrm{SrTiO}_{3}$, the mixed valence $\left(\mathrm{Fe}^{3+} / \mathrm{Fe}^{4+}\right)$ character of the dopant creates a charge imbalance with respect to the lattice, which is compensated by the creation of oxygen vacancies ${ }^{1}$ to conserve charge neutrality. Redox-type reactions of the crystal affect the balance between $\mathrm{Fe}^{3+}$ and $\mathrm{Fe}^{4+}$, and therefore the oxygen vacancy concentration in the material. It has been shown that such redox reactions, induced by thermal treatment under oxidizing or reducing atmosphere, influence the $\mathrm{X}$-ray absorption near-edge structure (XANES) of transition metal dopants, while the XANES of the Ti K-edge remains unchanged. ${ }^{2}$ The strong ionicity of the constituent species makes $\mathrm{SrTiO}_{3}$ susceptible to chemical changes induced by an electric field, ${ }^{3,4}$ resulting in a concentration polarization of oxygen vacancies that is visible as a color-change taking place at the anode and cathode (electrocoloration). ${ }^{5}$ This effect gained new interest in the last decade since the related insulator-to-metal

${ }^{a}$ Peter Grünberg Institute, Forschungszentrum Jülich, 52425 Jülich, Germany.E-mail: c.lenser@fz-juelich.de; Fax: + 492461612550

${ }^{b}$ Jülich-Aachen Research Alliance, Section Fundamentals of Future Information Technology (JARA-FIT), Germany

${ }^{c}$ Institute of Solid State Physics, University of Latvia,

Kengaraga Street 8, LV-1063 Riga, Latvia

${ }^{d}$ Institute of Physics, University of Silesia, 40-007 Katowice, Poland

${ }^{e}$ Institut für Werkstoffe der Elektrotechnik, RWTH Aachen,

52056 Aachen, Germany transition (electroformation) in $\mathrm{SrTiO}_{3}$ and other transition metal oxides can be exploited for the fabrication of non-volatile resistive random access memory (RRAM) devices. ${ }^{6-9}$ Microfocus $\mathrm{X}$-ray absorption spectroscopy ${ }^{8}$ at the K-edge of the Cr-dopant proved the redistribution of oxygen vacancies during the electroforming process in $\mathrm{SrTiO}_{3}$. However, despite of one singular report, ${ }^{10}$ the valence change in the Ti sublattice is generally insufficient to be detected at the Ti K-edge since oxygen vacancies are preferentially formed at the acceptor sites. ${ }^{8}$

The chemical equilibrium between charged defects poses an inherent challenge for XAFS investigations since it is almost impossible to obtain "pure" samples having only one type of defect. It is therefore necessary to deconvolute the measured signal using either appropriate standards or theoretical approximations. In addition, the absorption characteristics are strongly influenced by the polarized bonding environment in transition metal oxides. The connection between the formal valence state and the position and structure of the absorption edge is complicated by the fact that a preferred ground state configuration may be stabilized by a ligand-hole ${ }^{11}$ and by the influence of distortions in the coordination polyhedra, coordination number and site symmetry, all of which may be present in the probed volume.

In the present work, we investigate the valence state and the local environment of $\mathrm{Fe}$ in a $\mathrm{Fe}$-doped $\mathrm{SrTiO}_{3}$ single crystal after electrocoloration by a dc electric field using a combinatorial approach of three spectroscopic techniques. The concentration polarization associated with the electrocoloration creates an oxygen-rich region around the anode and a strongly 
oxygen-deficient region around the cathode. Using the fact that Raman spectroscopy and electron paramagnetic resonance (EPR) are sensitive only to a specific defect configuration of $\mathrm{Fe}$ in $\mathrm{SrTiO}_{3}$, it is the scope of this work to probe the respective defects using locally sensitive experimental techniques such as X-ray absorption spectroscopy. It should be noted that the concentration polarization induced by the dc electric field is only indirectly connected to the insulator-to-metal transition taking place locally confined between the electrodes. ${ }^{12}$

\section{Experiment}

A $0.2 \mathrm{wt} \% \mathrm{Fe}$-doped $\mathrm{SrTiO}_{3}$ single crystal was electrocolored using two Au-electrodes evaporated onto the (001)-oriented, polished crystal. A constant current was increased stepwise from $1 \mathrm{nA}$ to $0.1 \mathrm{~mA}$ over the duration of 2 weeks, until finally a breakdown in resistance occurred and a current of $10 \mathrm{~mA}$ flowed. The crystal was kept at a temperature of $300{ }^{\circ} \mathrm{C}$ at a vacuum pressure of $10^{-8} \mathrm{mbar}$. The coloration of the crystal is depicted in Fig. 1, along with an indication of the anodic and cathodic regions that were investigated in this work. After XAFS and Raman measurements, the crystal was cut into slices for the EPR spectrometer.

XAFS experiments were performed at beamlines BM29 (ESRF, Grenoble) and A1 (HASYLAB, Hamburg). X-Ray absorption near edge spectroscopy (XANES) and extended $\mathrm{X}$-ray absorption fine structure (EXAFS) were measured in fluorescence step-by-step (single crystal) and transmission mode (powder references) using monochromatic X-ray radiation, using a $\mathrm{Si}(111)$ double-crystal monochromator with an energy resolution of $\Delta E / E<10^{-4}$ at $7000 \mathrm{eV}$ photon energy. The size of the beam at the sample was typically $1 \times 8 \mathrm{~mm}^{2}$. XANES of the Fe K-edge (Ti K-edge) spectra were typically recorded within an interval of $100 \mathrm{eV}(50 \mathrm{eV})$ around the absorption edge, with a constant energy step of $0.5 \mathrm{eV}(0.2 \mathrm{eV})$. The Fe K- and Ti K-edge EXAFS spectra were recorded using an optimized energy mesh with a step of $0.2 \mathrm{eV}$ around the absorption edge and a constant $\Delta k$ step far above the edge.

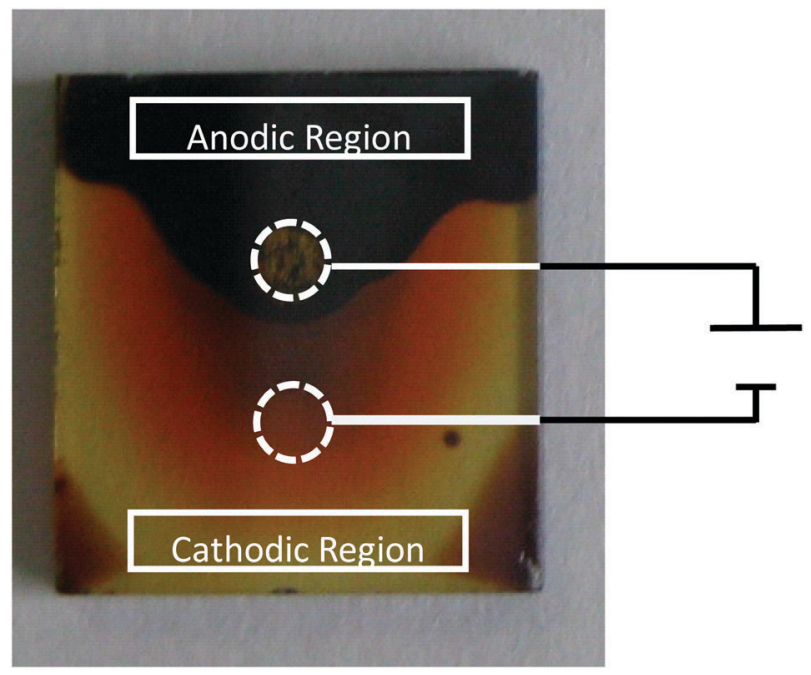

Fig. 1 Optical photograph of the electrocolored $\mathrm{Fe}$-doped $\mathrm{SrTiO}_{3}$ single crystal. The rectangles indicate the anodic and cathodic regions used in the analysis.
Micro-Raman spectra were collected at $20{ }^{\circ} \mathrm{C}$ using a confocal microscope with spectrometer Nanofinder-S (SOLAR TII, Ltd.). ${ }^{13}$ The measurements were performed through a Nikon Plan Fluor $40 \times(\mathrm{NA}=0.75)$ optical objective. The Raman spectra were excited by a $\mathrm{He}-\mathrm{Cd}$ laser $(441.6 \mathrm{~nm}, 50 \mathrm{~mW} \mathrm{cw}$ power) and dispersed by 600 grooves $\mathrm{mm}^{-1}$ diffraction grating mounted on the $520 \mathrm{~mm}$ focal length monochromator. A Peltier-cooled back-thinned CCD camera (ProScan HS-101H, $1024 \times 58$ pixels) was used as a detector. The elastic laser light component was eliminated by an edge filter (Omega, 441.6AELP-GP). Similar results (not shown here) were also obtained under excitation by a $532 \mathrm{~nm}$ DPSS solid state laser. The EPR spectra have been recorded with a X-band $(\nu=9.323 \mathrm{GHz})$ spectrometer with a modulation of $100 \mathrm{kHz}$. In order to study the angular dependence, thin slices of the Fe-doped single crystal were cut along the [010]-axis and aligned with [010] parallel to the rotation axis of the spectrometer. The dimensions of each piece were about $1 \times 10 \times 1 \mathrm{~mm}^{3}$. The samples were mounted on the $x y z$ orthogonal laboratory frame chosen for the EPR anisotropy pattern and related to the crystallographic axes as follows: $x \|$ [001], $z \|$ [010], $y \|$ [100]. The presented measurements were done at room temperature, while additional measurements at liquid nitrogen temperature confirm the presented results.

\section{Data analysis}

The XANES/EXAFS data treatment and fitting was done using the EDA software package. ${ }^{14}$ The XANES region of the absorption edge was extracted by background subtraction and normalization to $100 \mathrm{eV}$ above the edge. The EXAFS signal was extracted up to $k=10 \AA^{-1}$ after standard background subtraction, and the single-scattering contributions of the first shell were isolated by Back Fourier Transformation (BFT) in the interval $0.8-2.1 \AA$. The single-shell fitting of the EXAFS spectra was done using the single-scattering curved-wave formalism in $k$-space after BFT. In this paper, the EXAFS data were analyzed using two different approaches: the backscattering phases and amplitudes were either calculated by the FEFF8 $\operatorname{code}^{15}$ or obtained experimentally from the polycrystalline reference compound $\mathrm{LaFeO}_{3}$.

As the threshold energy of the photoelectron $E_{0}$ is defined in the FEFF8 code relative to the Fermi level and depends on the muffin-tin radii, the spectra have to be corrected to avoid $E_{0}$ difference errors in the fitting process. Consequently, the phase differences between theoretical and experimental spectra were set to zero at low $k$-values, according to Bunker and Stern's criterion, ${ }^{16}$ and $E_{0}$ was allowed to vary for fine adjustment during the fitting procedure.

The calculations by the FEFF8 code were performed for a cluster having a radius of $8 \AA$, which mimics the environment of the Fe ions in an $\mathrm{SrTiO}_{3}$ crystal (lattice parameter $a_{0}=3.905 \AA$ ) and centered on a Fe atom that has been substituted for Ti. A muffin-tin cluster potential was calculated in a self-consistent way, and the complex Hedin-Lundqvist exchange-correlation potential was used to account for inelastic effects (see the details in ref. 17). Experimental backscattering phases and amplitudes were extracted from the experimental EXAFS data measured on a polycrystalline $\mathrm{LaFeO}_{3}$ powder at room temperature. 
These functions were obtained assuming the iron coordination number $N=6$ and medium $\mathrm{Fe}-\mathrm{O}$ interatomic distance $R=$ $2.007 \AA$, from the crystallographic data, and the mean-square relative displacement (MSRD) Fe-O $\sigma^{2}=0.0033 \AA^{2}$. The MSRD value was obtained from the best fit of the Fe K-edge EXAFS signal for $\mathrm{LaFeO}_{3}$ using the single-shell Fe-O model with the theoretical phase and amplitude, calculated by the FEFF8 code for the $\mathrm{LaFeO}_{3}$ structure. ${ }^{18}$ While $\mathrm{LaFeO}_{3}$ is orthorhombic (space group Pnma), the distortion of the $\mathrm{Fe}-\mathrm{O}_{6}$ octahedra is smaller than the measurement uncertainty ( $\sim 0.005 \AA$ ), and therefore the first shell was treated as a perfect octahedron $\left(R_{\mathrm{Fe}-\mathrm{O}}=1.994 \pm 0.005 \AA\right)$. The good quality of the fit and the small MSRD obtained for the $\mathrm{Fe}-\mathrm{O}$ bond $\left(\sigma^{2}=0.0033 \AA^{2}\right)$ in $\mathrm{LaFeO}_{3}$ confirms the validity of this approach.

Note that the use of experimental phases and amplitudes for EXAFS allows, to a certain extent, the compensation of systematic errors as they include the contribution of the mean free path, the multi-electron amplitude reduction factor $S_{0}^{2}$, glitches and resolution, thus reducing the number of adjustable parameters and consequently increasing the reliability of the fitted results. To ensure the phases and amplitudes transferability, and to allow estimation of systematic errors, all the data were analyzed in a similar way, using also the theoretical phases and amplitudes, filtering procedures and parameters.

The fitting procedure was done using a parameter set of the disorder parameter MSRD $\sigma^{2}$ (describing the disorder in the bond length), the distance $R$, the amplitude reduction factor $N S_{0}^{2}$ and the energy shift $\Delta E_{0}$, in the case of the theoretical backscattering phases and amplitudes.

To provide a framework for the interpretation of the single crystal spectra, the EXAFS of powder references $\operatorname{SrTi}_{0.9} \mathrm{Fe}_{0.1} \mathrm{O}_{3}$ and $\mathrm{SrTi}_{0.9} \mathrm{Fe}_{0.1} \mathrm{O}_{2.95}$ were fitted as well. Note that the data on $\mathrm{SrTi}_{0.9} \mathrm{Fe}_{0.1} \mathrm{O}_{3}$ and $\mathrm{SrTi}_{0.9} \mathrm{Fe}_{0.1} \mathrm{O}_{2.95}$ calculated with the theoretical phase and amplitude were previously published. ${ }^{17}$ In the first step, the experimental spectrum of powder reference compound $\mathrm{LaFeO}_{3}$ was analyzed using the theoretically calculated scattering phase and amplitude. In the second step, all experimental spectra from powder references and the single crystal were treated with the experimental phase and amplitude extracted from the $\mathrm{LaFeO}_{3}$ spectra, as well as with theoretical phase and amplitude.

Self-consistent, real-space full-multiple-scattering XANES calculations of the Fe K-edge in Fe-doped $\mathrm{SrTiO}_{3}$ have been performed by the FDMNES code ${ }^{19,20}$ in the one-electron electric dipole $(\Delta l=1,1 s \rightarrow \mathrm{n} p)$ and quadrupole $(\Delta l=2$, $1 s \rightarrow \mathrm{n} d$ ) approximations. We considered four structural models based on the clusters, centered at the absorbing $\mathrm{Fe}$ atom and constructed from the cubic $\mathrm{SrTiO}_{3}$ crystal structure (lattice parameter $a_{0}=3.905 \AA$ ). The radius of the clusters was equal to $6 \AA$, being sufficiently large to reproduce the main features in the experimental Fe K-edge XANES signal. In the first model $\left(\mathrm{SrTiO}_{3}: \mathrm{Fe}\right)$, the substitution of one Ti atom by the $\mathrm{Fe}$ atom was performed. In the second model $\left(\mathrm{SrTiO}_{3}: \mathrm{Fe}\right.$ with JT), the Jahn-Teller (JT) distortion ${ }^{17}$ of the first coordination shell of the absorbing iron atom was taken into account: the $\left[\mathrm{FeO}_{6}\right]$ octahedron was distorted by placing four in-plane oxygen atoms at $1.89 \AA$ from iron and two oxygen atoms at $2.10 \AA$. In the third model $\left(\mathrm{SrTiO}_{3}: \mathrm{Fe}+\mathrm{V}_{\mathrm{O}}\right)$, no JT distortion was present, but an oxygen vacancy $\left(\mathrm{V}_{\mathrm{O}}\right)$ was introduced into the first coordination shell of the Fe atom, i.e. one of the six oxygen atoms was eliminated, so that the iron atom becomes five-fold coordinated. Finally, in the fourth model $\left(\mathrm{SrTiO}_{3}\right.$ : $\mathrm{Fe}+\mathrm{V}_{\mathrm{O}}^{2 \text { shell }}$ ), no JT distortion was present, but an oxygen vacancy $\left(\mathrm{V}_{\mathrm{O}}\right)$ was introduced into the second coordination shell of the Fe atom. The self-consistent cluster potential was calculated within the non-muffin-tin approximation using the finite difference method (FDM) ${ }^{20}$ in which the full cluster potential is set on an equally spaced 3D-grid. The real Hedin-Lundqvist exchange-correlation potential was employed in the calculations, and the final excited state of the photoelectron included a screened core-hole. Note that the calculated XANES signals were intentionally not broadened to underline the fine structure details.

\section{Results and discussion}

The generally accepted model of electrocoloration in Fe-doped $\mathrm{SrTiO}_{3}$ is based on the field-driven migration of oxygen vacancies and the corresponding chemical changes at the Fe centers that give rise to the color change. ${ }^{5}$ In the low concentration (dilute) regime where the Fe centers can be considered as isolated, a set of equations can be used to describe the defect equilibria in the ionic crystal. Of special interest for this work are the conservation conditions: ${ }^{1}$

$$
\begin{gathered}
{[\mathrm{Fe}]_{\text {tot }}=\left[\mathrm{Fe}^{3+}\right]+\left[\mathrm{Fe}^{4+}\right]} \\
2\left[\mathrm{~V}_{\ddot{\mathrm{O}}}\right]+[h]=\left[\mathrm{Fe}^{3+}\right]+[e] .
\end{gathered}
$$

In particular, the weak band-gap excitation (no free charge carriers) allows the simplification of eqn (2):

$$
2\left[\mathrm{~V}_{\ddot{\mathrm{O}}}\right] \approx\left[\mathrm{Fe}^{3+}\right] \text {. }
$$

This charge neutrality condition imposes some constraints on the defect structure that can be present in the material. Most notably, even under completely reduced conditions, eqn (3) demands that the oxygen vacancy concentration will not be more than half of the total Fe concentration, assuming that every $\mathrm{Fe}$ center has been reduced to $\mathrm{Fe}^{3+}$ and that other mechanisms of charge compensation can be neglected. For strongly reduced crystals, the charge neutrality condition has to be expanded to accommodate the compensation of oxygen vacancies by free electrons, ${ }^{21}$ causing the appearance of $\mathrm{Ti}^{3+}$ ions through the occupation of conduction band states. To verify the validity of eqn (3) for the present work, it is necessary to monitor the chemical changes induced by the electrocoloration at both the Fe- and Ti-site. Since there is no observable change in the Ti K-edge XANES (Fig. 2), and the average Ti-O bond distance as extracted from EXAFS data is identical in the anodic and cathodic regions (within the measurement uncertainty, data not shown), it follows that the oxidation/reduction process takes place preferentially at the Fe-site and that the changes at the Ti-site are too low to be detectable.

Therefore, the following sections are concerned with the structural and chemical changes at the Fe-site induced by the electrocoloration process, and eqn (3) will be regarded as valid.

\subsection{EPR studies}

Fig. 3 shows the EPR spectra of $\mathrm{Fe}^{3+}$ of the cathodic (upper, red line) and anodic regions (lower, black line) of the doped 


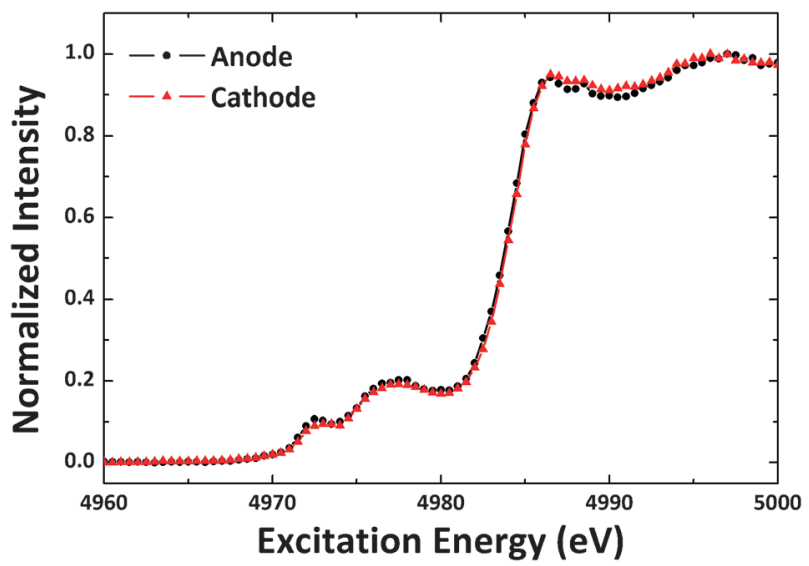

Fig. 2 XANES of the Ti K-edge from the anodic (black circles) and cathodic (red triangles) regions of the electrocolored Fe-doped $\mathrm{SrTiO}_{3}$ single crystal. No noticeable changes are observed in the edge structure indicating that the oxidation state of $\mathrm{Ti}$ atoms remains unchanged upon the electrocoloration process.

$\mathrm{SrTiO}_{3}$ single crystal, with the static magnetic field parallel to the crystallographic [010] direction. The EPR signal in the anodic region consists of a strong, isotropic central line, with 4 symmetrically split satellite lines showing an angular variation identical to that found in ref. 22, with the isotropic $g$-factor of 2.00 corresponding to cubic $\mathrm{Fe}^{3+}$ centers and with a cubic splitting parameter of $a \approx 0.02 \mathrm{~cm}^{-1}$.

The EPR signal of the cathodic region consists of two structurally non-equivalent centers, a cubic center equivalent to that in the anodic region and an axial center with a strongly anisotropic $g$-factor, as is well described in the literature. ${ }^{23,24}$ Kirkpatrick et al. ${ }^{23}$ found these lines with $g_{\perp}=5.993$ and $g_{\|}=2.0054$ to correspond to the $-1 / 2 \leftrightarrow 1 / 2$ transition of $\mathrm{Fe}^{3+}$ in an axial site with a strong tetragonal distortion. No other lines are visible due to the strong zero-field splitting induced by the local crystal field. The angular variation of the effective $g$-factor of the axial center is shown in Fig. 4, reproducing the angular dependence demonstrated by Kirkpatrick et al. remarkably well.

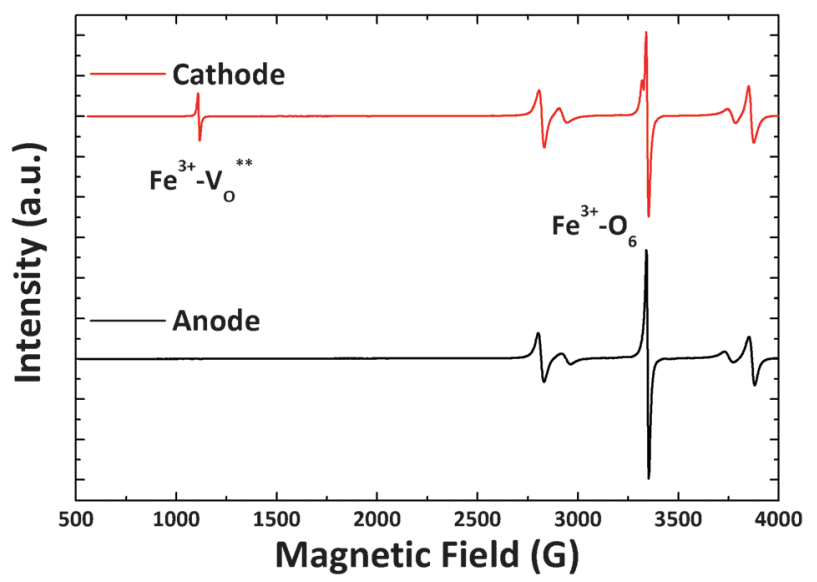

Fig. 3 EPR signals of $\mathrm{Fe}^{3+}$ in the anodic (lower, black line) and cathodic (upper, red line) regions of the electrocolored Fe-doped $\mathrm{SrTiO}_{3}$ single crystal. The relative amplitude of the EPR signals is normalized to the maximum. Note that the intensity of the normalized spectra is not comparable.

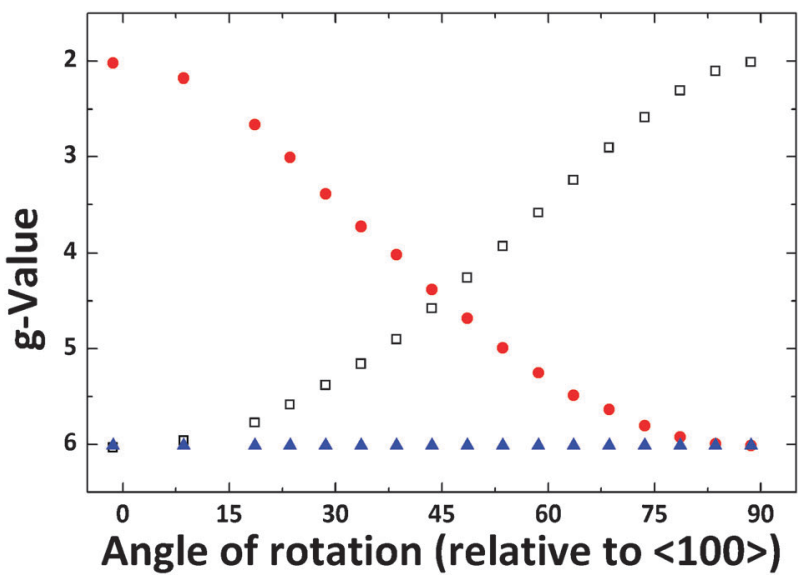

Fig. 4 Angular dependence of the effective $g$-value of the axial $\mathrm{Fe}^{3+}$ center in the cathodic region. The angle of rotation corresponds to the angle between the magnetic field lines and the crystallographic [100] axis.

We can conclude that after the electrocoloration process, the axial $\mathrm{Fe}^{3+}$ center in the anodic part has disappeared almost completely, while the signal of the cubic $\mathrm{Fe}^{3+}$ center is decreased by about a factor of $7( \pm 1)$ with respect to the cathodic part. It should be mentioned that the $\mathrm{Fe}^{4+}$ center created in the anodic region is a "non-Kramers" ion due to the large contribution of the $d^{4}$ configuration to the ground state, and therefore does not appear in the spectra. ${ }^{25}$ There are no other EPR-active impurity centers with significant contributions to the EPR spectra.

\subsection{Raman spectroscopy}

Since $\mathrm{SrTiO}_{3}$ at room temperature has a cubic structure (space group $P m \overline{3} \mathrm{~m}$ ) with a center of inversion, the undoped crystal exhibits no first-order Raman scattering, and only secondorder effects are observed in the Raman spectra. An extensive examination of the Raman spectra of oxidized and reduced $\mathrm{SrTi}_{1-x} \mathrm{Fe}_{x} \mathrm{O}_{3-\delta}$ solid solution powders has been given in ref. 17, and the existence of a first-order Raman peak at $690 \mathrm{~cm}^{-1}$ has been attributed to doping with $\mathrm{Fe}^{4+}$ ions. In addition, three "forbidden" phonon modes have been weakly visible: LO3, TO4 and LO4 at $475 \mathrm{~cm}^{-1}, 545 \mathrm{~cm}^{-1}$ and $795 \mathrm{~cm}^{-1}$, respectively. On the other hand, $\mathrm{Fe}^{3+}$ does not induce any change in the Raman spectrum with regard to that of an undoped crystal. Therefore, the appearance of the strong peak at $690 \mathrm{~cm}^{-1}$ can be seen as a "fingerprint" indicating the presence of $\mathrm{Fe}^{4+}$ in the crystal.

In the present work, Raman spectroscopy was performed close to the anode and cathode of the Fe-doped $\mathrm{SrTiO}_{3}$ single crystal, using a confocal Raman microscope with a spatial resolution of $1 \mu \mathrm{m}$. The recorded spectrum (Fig. 5) of the anodic (solid black line) region shows the distinct features that characterize the oxidized $\mathrm{SrTi}_{1-x} \mathrm{Fe}_{x} \mathrm{O}_{3}$ powders as described above. In the spectrum of the cathodic region (dashed red line), the additional peak as well as the weak phonon modes are notably absent, indicating that the amount of $\mathrm{Fe}^{4+}$ in the cathodic region is too low to be detected by Raman spectroscopy. From the Raman data, we can conclude that the cathodic region does not contain significant amounts of $\mathrm{Fe}^{4+}$, while the anodic region contains predominantly $\mathrm{Fe}^{4+}$. 


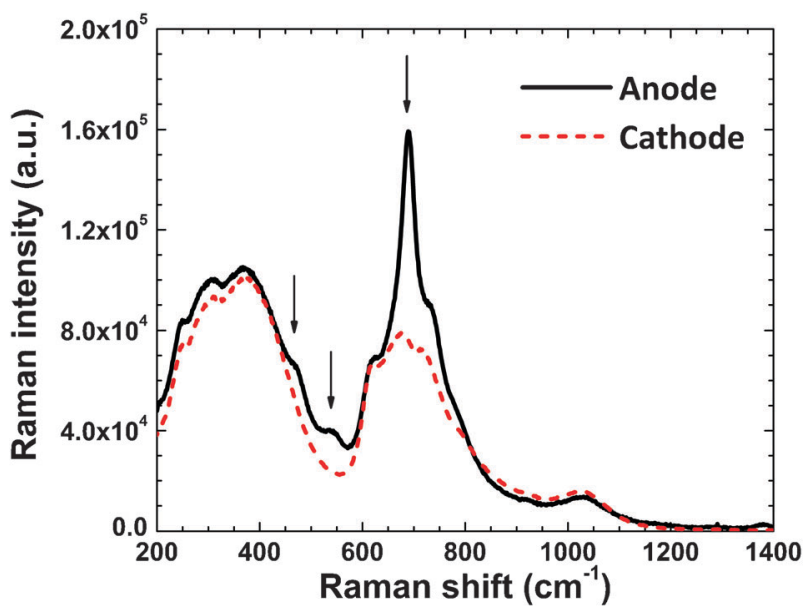

Fig. 5 Raman scattering spectra from the anodic (solid black line) and cathodic (dashed red line) regions of the electrocolored Fe-doped $\mathrm{SrTiO}_{3}$ single crystal. Note the developing shoulders and the pronounced peak indicated by the arrows.

\subsection{XANES studies}

A comparison of the experimental Fe K-edge XANES for the cathodic and anodic regions in the Fe-doped $\mathrm{SrTiO}_{3}$ single crystal (Fig. 6(a)) indicates that while the pre-edge peak located at $7115 \mathrm{eV}$ is similar in both cases, the fine structure located at higher energies is different. In particular, the shoulder at $7123 \mathrm{eV}$ is more pronounced in the cathodic region, and the main absorption edge shifts by about $\sim 0.5 \mathrm{eV}$ to higher energies in the anodic region. Reference XANES data (from ref. 17) of completely oxidized and reduced $\mathrm{SrTi}_{1-x} \mathrm{Fe}_{x} \mathrm{O}_{3-\delta}$ solid solution powders show an almost identical shift of the main absorption edge (Fig. 6(b)). Comparing the XANES data for the single crystal to the reference data, it is obvious that the changes taking place during electrocoloration affect the valence state of the $\mathrm{Fe}$ atoms in a reduction/oxidation process, leaving $\mathrm{Fe}$ centers predominantly oxidized in the anodic region and reduced in the cathodic region.

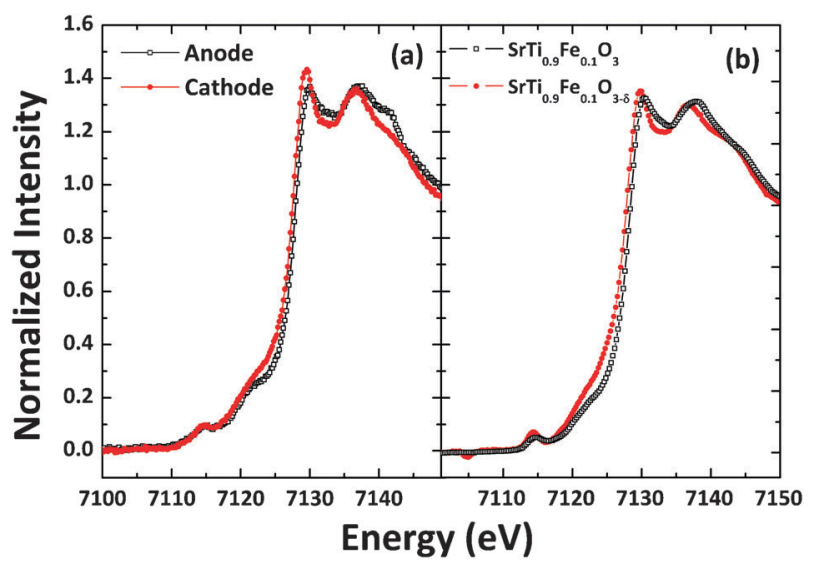

Fig. 6 (a) Fe K-edge XANES spectra from the anodic (black, open squares) and cathodic (red, solid circles) regions of the electrocolored Fe-doped $\mathrm{SrTiO}_{3}$ single crystal. (b) Fe K-edge XANES spectra of powder solid solution $\mathrm{SrTi}_{0.9} \mathrm{Fe}_{0.1} \mathrm{O}_{3}$, fully oxidized (black, open squares) and reduced (red, solid circles) (data from ref. 17).

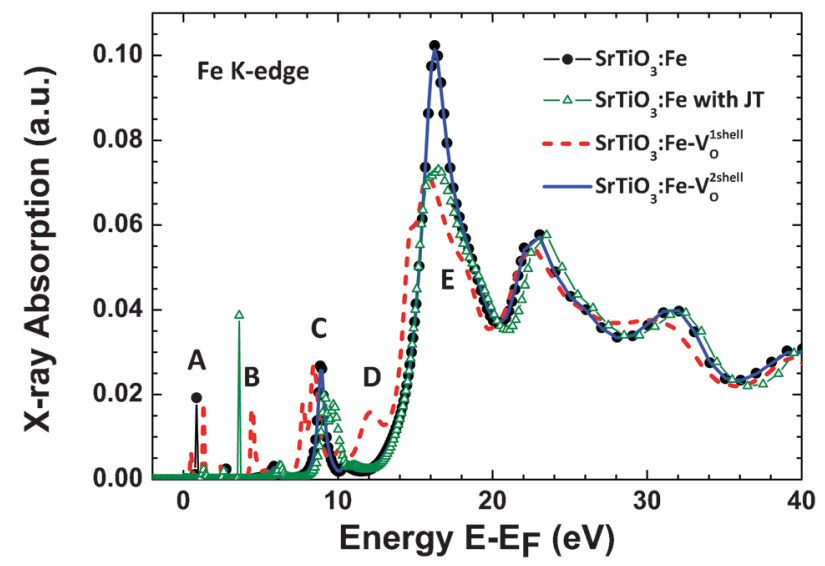

Fig. 7 Comparison of the calculated $\mathrm{Fe}$ K-edge XANES for $\mathrm{Fe}$-doped $\mathrm{SrTiO}_{3}$ having no oxygen vacancy (solid, black circles and open, green triangles) and with a single oxygen vacancy $V_{O}$ in the first (dashed, red line) or second (solid, blue line) coordination shell of iron. In the absence of oxygen vacancies, the two models correspond to (i) the unrelaxed octahedral iron environment (solid, black circles) and (ii) the relaxed $\mathrm{FeO}_{6}$ octahedron due to the Jahn-Teller distortion ${ }^{26}$ (open, green triangles). The zero of the energy scale corresponds to the theoretical Fermi level $E_{\mathrm{F}}$. Calculations were performed taking into account the dipole and quadrupole transitions.

Deeper understanding of the Fe K-edge XANES signals has been obtained using theoretical simulations, performed for the four models as described in Section 3. The results of the XANES calculations are shown in Fig. 7 and are compared with the experimental data for the cathodic and anodic regions in Fig. 8. There are four features (A, B, C, D) in the XANES signals that can be distinguished below the main absorption edge $\mathrm{E}$. At the Fe K-edge, the $1 s(\mathrm{Fe})$ core-electron is excited, following dipole selection rules, to empty final-states with $p$-character, located above the Fermi level and being relaxed due to the presence of the positively charged core-hole. Such transitions give rise to peaks starting from $\mathrm{B}$ and above (Fig. 7). The transition of the $1 s$ electron to the conduction band of cubic Fe-doped $\mathrm{SrTiO}_{3}$ (peak A in Fig. 7), composed mainly of the $3 d$-states of the $\mathrm{Ti}(\mathrm{Fe})$ ions, is forbidden in the dipole approximation. However, it becomes allowed through symmetry lowering due to the Jahn-Teller distortion, ${ }^{26}$ the presence of an oxygen vacancy in the first coordination shell of iron or within the quadrupole approximation. Note that generally, the small cross section of the $1 s \rightarrow 3 d$ quadrupole transition is compensated by a high density of $3 d$ states.

Next we will discuss the peculiarities of the Fe K-edge XANES signals for the four theoretical models (Fig. 7). By looking at the first and second models, corresponding to the regular and JT-distorted iron environments, respectively, one can see that the presence of the JT distortion leads mainly to a decrease of the main peak $E$ amplitude and a splitting of peak C. Thus the JT distortion cannot explain an increase of the absorption in the energy range from 10 to $15 \mathrm{eV}$ and, also, the origin of the shoulder around $30 \mathrm{eV}$ in the experimental data (Fig. 8).

Next two models take into account the possible presence of the oxygen vacancy $\left(\mathrm{V}_{\mathrm{O}}\right)$ in the first or second coordination shell of iron. Contrary to the case of the JT distortion, an 

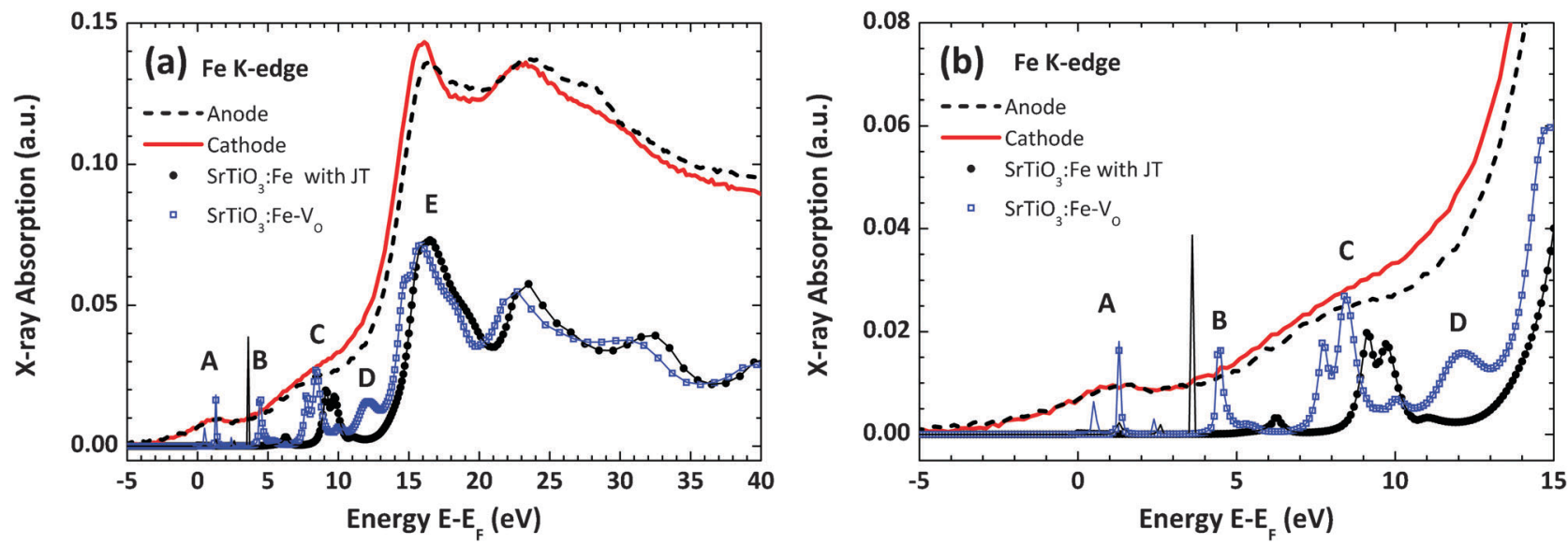

Fig. 8 Comparison of the experimental and calculated Fe K-edge XANES in Fe-doped $\mathrm{SrTiO}_{3}$. The experimental XANES correspond to the cathode (solid red line) and anode (dashed black line) regions of the electrocolored Fe-doped $\mathrm{SrTiO}_{3}$ single crystal. The calculations were performed in the dipole approximation for the two models: JT-distorted iron environment without oxygen vacancies (solid, black circles) and with a single oxygen vacancy $\mathrm{V}_{\mathrm{O}}$ in the first coordination shell of unrelaxed iron environment (open, blue squares). The zero of the energy scale corresponds to the theoretical Fermi level $E_{\mathrm{F}}$. Four features (A, B, C, D), located in the pre-edge region, and the main peak E are indicated. In addition, the results for calculations in the dipole plus quadrupole approximation are shown as solid lines, being completely identical to the dipole approximation except for the range of feature $\mathrm{A}(0-4 \mathrm{eV})$.

introduction of the single oxygen vacancy into the first coordination shell of iron (the third model) makes the theoretical Fe K-edge XANES signal closer to the experimental one (Fig. 8). The most prominent effect is observed in the range of 4-12 eV above the Fermi level. Here two new peaks B and D appear, and peak $\mathrm{C}$ becomes split. The position of peak $\mathrm{D}$ corresponds well to the energy region, where the experimental XANES signals for the cathodic and anodic regions differ. Therefore, the occurrence of peak D can be used as a "fingerprint" to trace the presence of an oxygen vacancy in the first coordination shell of iron.

Note that placing the single oxygen vacancy into the second coordination shell of iron (the fourth model) results in the $\mathrm{Fe}$ K-edge XANES signal being very similar to that without vacancy (Fig. 7). Such result can be expected since there are 24 oxygen atoms in the second shell, so that the absence of one atom produces negligible influence on the total XANES signal.

To conclude, we attribute a variation of the absorption intensity observed between the two experimental Fe K-edge XANES signals from the anodic and cathodic regions of the $\mathrm{Fe}$-doped $\mathrm{SrTiO}_{3}$ single crystal to the appearance of an oxygen vacancy in the first coordination shell of Fe. Our interpretation of the Fe K-edge XANES in Fe-doped $\mathrm{SrTiO}_{3}$ agrees with that by Janousch et al. ${ }^{8}$ for $\mathrm{Cr}$-doped $\mathrm{SrTiO}_{3}$, where it was shown, using density functional theory (DFT) calculations, that the presence of an oxygen vacancy in the octahedron surrounding the chromium atom leads to an increase of the $\mathrm{Cr} 4 \mathrm{p}$ density of states and, thus, increases absorption in the chromium K-edge XANES below the main absorption edge.

\subsection{EXAFS studies}

The experimental EXAFS $\chi(k) k$ spectra for the anodic and cathodic regions are shown in Fig. 9. Their Fourier transforms (FTs), shown in Fig. 10, indicate a change of the first peak position (better seen in the imaginary part of FTs) at $\sim 1.4 \AA$ due to the first coordination shell of iron. At the same time,

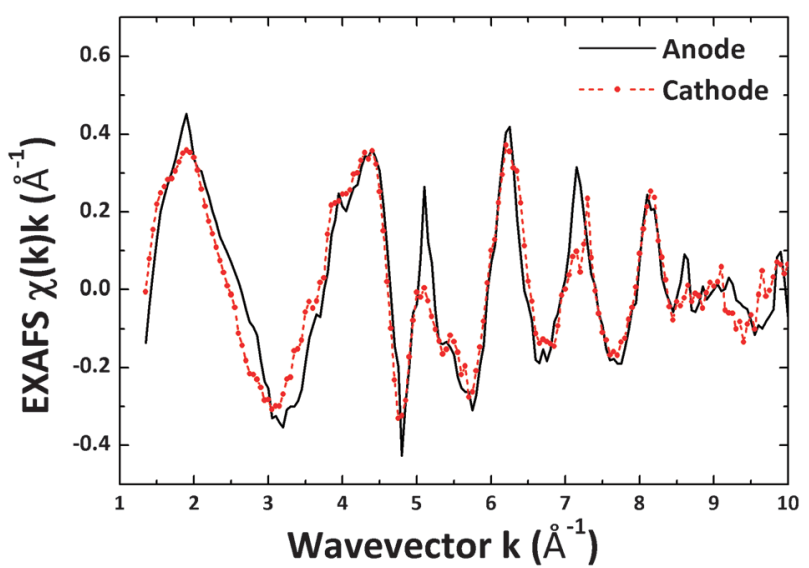

Fig. 9 Experimental Fe K-edge EXAFS spectra from the cathode (solid, black line) and anode (dashed, red line) regions of the electrocolored $\mathrm{Fe}$-doped $\mathrm{SrTiO}_{3}$ single crystal.

the position of the second and third coordination shells (peaks in the region 2.5-4 $\mathrm{A}$, Fig. 10) remains unchanged. This suggests that the electrocoloration process induces a difference in the average $\mathrm{Fe}-\mathrm{O}$ bond distance in the anodic and cathodic regions of the single crystal.

The fitting results obtained (as described in Section 3) with the experimental phase and amplitude are presented in Table 1. As one can expect from the Shannon radii of iron ions $\left(r\left(\mathrm{Fe}^{3+}\right)=0.645 \AA\right.$, $\left.r\left(\mathrm{Fe}^{4+}\right)=0.565 \AA\right)$, the $\mathrm{Fe}^{3+}-\mathrm{O}$ bond length as extracted from EXAFS of $\mathrm{Fe}^{3+}-\mathrm{O}$ in $\mathrm{LaFeO}_{3}$ $(1.994 \pm 0.005 \AA)$ is significantly longer than that of $\mathrm{Fe}^{4+}-\mathrm{O}$ in $\mathrm{SrFeO}_{3}(1.925 \AA) .{ }^{27}$ Using the experimental phase and amplitude for the fitting procedure, we observe that the $\mathrm{Fe}-\mathrm{O}$ bond distance in the anodic part of the crystal $(1.932 \AA)$ is very close to the distance of the fully oxidized $\mathrm{SrTi}_{0.9} \mathrm{Fe}_{0.1} \mathrm{O}_{3}$ powder reference $(1.931 \AA)$. In addition, the large MSRD value for both spectra is characteristic of the Jahn-Teller distortion around 


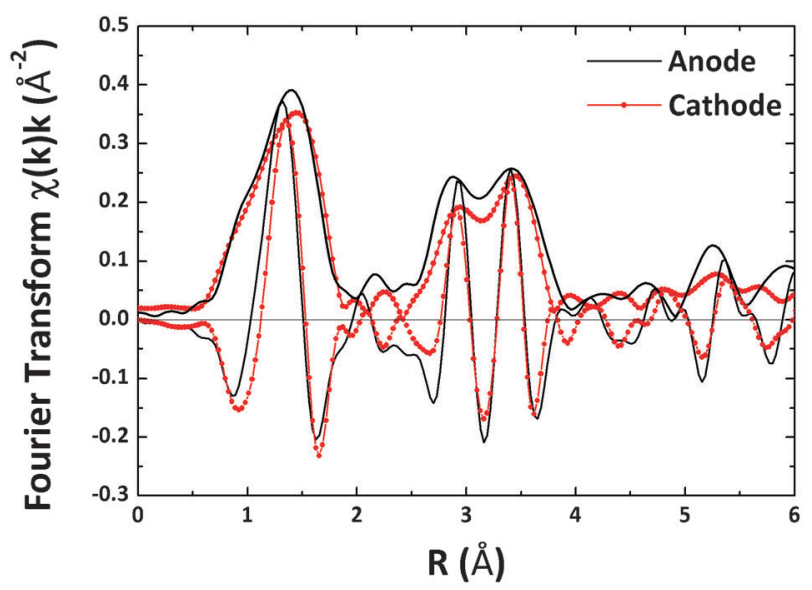

Fig. 10 Magnitude and imaginary part of the Fourier transform of the Fe K-edge EXAFS signals shown in Fig. 9 for the anodic (solid, black lines) and cathodic (red circles) regions of the electrocolored Fe-doped $\mathrm{SrTiO}_{3}$ single crystal. The shift of the first peak at $\sim 1.4 \AA$, corresponding to the first coordination shell of iron (the $\mathrm{Fe}-\mathrm{O}$ distance), is clearly visible.

$\mathrm{Fe}^{4+}\left(d^{4}\right.$ configuration $)$ in $\mathrm{SrTiO}_{3} \cdot{ }^{17}$ From the EXAFS and XANES analyses as well as Raman, it follows that $\mathrm{Fe}$ is predominantly tetravalent in the anodic region, albeit with a small admixture of $\mathrm{Fe}^{3+}$ (cubic center, $d^{5}$ configuration) as shown by EPR (see Section 4.1).

In contrast, the $\mathrm{Fe}-\mathrm{O}$ bond length in the cathodic part $(1.972 \AA)$ is 4 pm longer than in the anodic region, while the MSRD decreases significantly. The EPR and Raman results show the existence of cubic $\mathrm{Fe}^{3+}$ centers in this region, with an admixture of $\mathrm{Fe}^{3+}-\mathrm{V}_{\ddot{O}}$ complexes, and no $\mathrm{Fe}^{4+}$. The $\mathrm{Fe}^{3+} \mathrm{O}$ distance in reduced $\mathrm{SrTi}_{0.9} \mathrm{Fe}_{0.1} \mathrm{O}_{2.95}(1.955 \AA)$ is intermediate between that in $\operatorname{SrTi}_{0.5} \mathrm{Fe}_{0.5} \mathrm{O}_{2.5}(1.960 \AA)^{17}$ and the $\mathrm{Ti}-\mathrm{O}$ distance in $\mathrm{SrTiO}_{3}(1.951 \AA$ ), while the $\mathrm{Fe}-\mathrm{O}$ distance in the cathodic region of the single crystal is significantly longer. In addition, the slightly larger MSRD value observed in $\mathrm{SrTi}_{0.9} \mathrm{Fe}_{0.1} \mathrm{O}_{2.95}$ compared with the cathodic part suggests a larger distortion in the first coordination shell around $\mathrm{Fe}^{3+}$ in the reduced solid solution.

\section{Discussion}

The experimental determination of the $\mathrm{Fe}^{3+}-\mathrm{V}_{\ddot{O}}$ concentration has been shown to be possible via double-integration EPR techniques ${ }^{24}$ and also attempted via linear combination of XANES spectra. ${ }^{8}$ In principle, the relative concentrations of Fe centers can be estimated from linear combination of the $\mathrm{Fe}-\mathrm{O}$ bond distances using suitable standards. Care has to be taken to determine which type of Fe center is present in the analyzed sample. Following the EPR and Raman results, we can assume that the only defect centers present in the anodic region of the single crystal are 6-fold coordinated $\mathrm{Fe}^{3+}$ and $\mathrm{Fe}^{4+}$ without any $\mathrm{Fe}^{3+}-\mathrm{V}_{\ddot{\mathrm{O}}}$. Suitable standards are $\mathrm{LaFeO}_{3}$ and $\mathrm{SrFeO}_{3}$ with $\mathrm{Fe}-\mathrm{O}$ distances of $1.994 \AA$ and $1.925 \AA$, respectively. For these compounds, it has been shown that A-site substitution of $\mathrm{Sr}$ for La decreases the size of the unit cell, despite the smaller Shannon radius of the $\mathrm{La}^{3+}$ ion, and the $\mathrm{Fe}-\mathrm{O}$ bond distance has been shown to decrease continuously with increasing amount of $\mathrm{Fe}^{4+}$ going from $\mathrm{LaFeO}_{3}$ to $\mathrm{SrFeO}_{3}$ without systematic deviations from the Vegard's law. ${ }^{28}$ Assuming a linear relation of the $\mathrm{Fe}-\mathrm{O}$ distance to concentration, the $\mathrm{Fe}^{3+}$ concentration in the anodic part can be estimated to be approximately $10 \%$. Integrating the EPR signals, we can further estimate the ratio (cathode to anode) of $\mathrm{Fe}^{3+}$ to be about $7( \pm 1)$, which puts the concentration of the cubic $\mathrm{Fe}^{3+}-\mathrm{O}_{6}$-center at roughly $70 \%( \pm 10 \%)$ in the cathodic region. Since Raman spectroscopy demonstrates the absence of $\mathrm{Fe}^{4+}$ in this region, consequently the amount of $\mathrm{Fe}^{3+}-\mathrm{V}_{\ddot{O}}$ can be estimated as $30 \%$. Unfortunately, the above estimation cannot be used to gain information on the $\mathrm{Fe}-\mathrm{O}$ bond length in the $\mathrm{Fe}^{3+}-\mathrm{V}_{\ddot{\mathrm{O}}}$ complexes because of the tetragonal distortion of the axial site.

Merkle and Maier ${ }^{24}$ demonstrate that the degree of association of Fe and oxygen vacancies shows a tendency to decrease with increasing doping level and temperature. For a similar doping concentration as used in this study, the degree of association at room temperature is given as $\sim 25 \%$, while values approaching $50 \%$ are only seen for lower concentrations and temperatures below $273 \mathrm{~K}$. Since these values are in agreement within the error bar specified above, a $\mathrm{Fe}^{3+}-\mathrm{V}_{\ddot{O}}$ concentration of $30 \%$ is a realistic estimation.

From the EXAFS and XANES analysis as well as from EPR data, it is reasonable to conclude that Fe exists mainly as a cubic $\mathrm{Fe}^{3+}$ center with $\mathrm{Fe}-\mathrm{O}$ distance $R=1.972 \AA$ in the cathodic region of the single crystal. The concentrations of the cubic $\mathrm{Fe}^{3+}-\mathrm{O}_{6}$ and the axial $\mathrm{Fe}^{3+}-\mathrm{V}_{\ddot{\mathrm{O}}}$ center are in agreement with the defect chemical constraint $\left[\mathrm{V}_{\mathrm{O}}\right] /\left[\mathrm{Fe}^{3+}\right]<0.5$ for the dilute regime (eqn (3), Section 4), with the amount of the $\mathrm{Fe}^{3+}-\mathrm{V}_{\ddot{O}}$ present around the cathode estimated to be $\sim 30 \%$. As $\operatorname{SrTi}_{0.9} \mathrm{Fe}_{0.1} \mathrm{O}_{2.95}$ is far outside the dilute regime, ${ }^{1}$ the constraints described by eqn (3) are no longer valid and $\mathrm{Fe}^{3+}-\mathrm{V}_{\ddot{O}}$ with an $\mathrm{Fe}-\mathrm{O}$ distance of $R=1.955 \AA$ becomes the predominant Fe center.

\section{Summary and conclusions}

In this work the electrochemically induced changes in the local structure and valence state of iron atoms have been studied in electrocolored $\mathrm{Fe}$-doped $\mathrm{SrTiO}_{3}$ single crystals by EPR, Raman scattering and X-ray absorption (XANES and EXAFS)

Table 1 Best-fit structural parameters $\left(N\right.$ is the coordination number, $R$ is the interatomic distance, and $\sigma^{2}$ is the mean-square relative displacement (MSRD)) for the first coordination shell of iron obtained from the analysis of the Fe K-edge EXAFS spectra using experimental backscattering phase and amplitude functions extracted from $\mathrm{LaFeO}_{3}$

\begin{tabular}{lllll}
\hline & $\mathrm{SrTi}_{0.9} \mathrm{Fe}_{0.1}^{4+} \mathrm{O}_{3}$ & $\mathrm{SrTi}_{0.9} \mathrm{Fe}_{0.1}^{3+} \mathrm{O}_{2.95}$ & $\mathrm{Fe}: \mathrm{SrTiO}_{3}$ (anode) & Fe:SrTiO \\
\hline$N$ & $5.4 \pm 0.1$ & $5.0 \pm 0.1$ & $5.7 \pm 0.1$ & $5.4 \pm 0.1$ \\
$R(\mathrm{Fe}-\mathrm{O}) / \AA$ & $1.931 \pm 0.005$ & $1.955 \pm 0.005$ & $1.932 \pm 0.005$ & $1.972 \pm 0.005$ \\
$\sigma^{2}(\mathrm{Fe}-\mathrm{O}) / \AA^{2}$ & $0.0094 \pm 0.0005$ & $0.0072 \pm 0.0005$ & $0.0098 \pm 0.0005$ & $0.0056 \pm 0.0005$ \\
\hline
\end{tabular}


spectroscopy. With EPR spectroscopy, we demonstrate the presence of axial $\mathrm{Fe}^{3+}-\mathrm{V}_{\mathrm{O}}$ centers in the cathodic region, with the characteristic angular dependence of the axial center, although the majority of $\mathrm{Fe}$ centers in the cathodic region are cubic $\mathrm{Fe}^{3+}$ centers. The anodic region of the electrocolored crystal is highly oxidized since the signal associated with the $\mathrm{Fe}^{3+}-\mathrm{V}_{\ddot{O}}$ complexes vanishes almost completely. Since EPR is not sensitive to $\mathrm{Fe}^{4+}$ ions ( $d^{4}$ configuration), Raman spectroscopy complements the EPR technique as the Raman line at $690 \mathrm{~cm}^{-1}$ is a "fingerprint" of $\mathrm{Fe}^{4+}$ in $\mathrm{Fe}$-doped $\mathrm{SrTiO}_{3}$. The strong appearance of this line in the anodic part is due to the predominance of $\mathrm{Fe}^{4+}$ in this region, whereas its disappearance in the cathodic part demonstrates the absence of $\mathrm{Fe}^{4+}$.

As shown by $a b$ initio full-multiple-scattering XANES calculations, a variation of the experimental Fe K-edge XANES observed between the anodic and cathodic regions can be attributed to the change in the amount of oxygen vacancies in the first coordination shell of iron atoms, in agreement with the EPR results.

EXAFS analysis yields a significant increase of the average $\mathrm{Fe}-\mathrm{O}$ distance by $\sim 0.03-0.04 \AA$ in the cathodic region, compared to both the anodic region and the $\mathrm{SrTi}_{0.9} \mathrm{Fe}_{0.1}^{4+} \mathrm{O}_{3}$ powder reference. By combination of EPR and EXAFS, we show that this increase is caused mainly by the presence of cubic $\mathrm{Fe}^{3+}$ centers in the cathodic region, whereas the smaller $\mathrm{Fe}-\mathrm{O}$ distance in the powder can be attributed to a much higher concentration of oxygen vacancies compared to the single crystal. The shorter $\mathrm{Fe}-\mathrm{O}$ distance in the anodic part is characteristic of $\mathrm{Fe}^{4+}$ centers, in good agreement with the $\mathrm{SrTi}_{0.9} \mathrm{Fe}_{0.1} \mathrm{O}_{3}$ powder reference and observations by Raman spectroscopy.

In the dilute regime, the Fe-centers can be considered as isolated and non-interacting, and the large $\mathrm{Fe}^{3+}-\mathrm{O}$ bond length is evidence of a local expansion of the crystal lattice around cubic Fe-centers $\left(\mathrm{Fe}^{3+}-\mathrm{O}_{6}\right)$. This suggests that the formation of $\mathrm{Fe}^{3+}-\mathrm{V}_{\mathrm{O}}$ complexes is favored by the minimization of strain energy in the crystal in addition to the association enthalpy. ${ }^{24}$ Above the dilute limit, the reduced distance between Fe-centers provides some strain relaxation which results in smaller $\mathrm{Fe}-\mathrm{O}$ distances even in the reduced material. ${ }^{17}$ These considerations enable the determination of the Fe valence state in the dilute regime based on the X-ray absorption characteristics.

\section{Acknowledgements}

This work has been performed within the EC FP7 MATERA project "Functional materials for resistive switching memories" (FMRSM). The authors acknowledge financial support by the NRW-EU Ziel 2-Programm. The research leading to these results has received funding from the European Community's Seventh Framework Programme (FP7/2007-2013) under grant agreement No. 226716 (Project I-20090034 EC). A.K. and J.P. were supported by the ESF and ERAF Projects 2009/ 0202/1DP/1.1.1.2.0/09/APIA/VIAA/141 and 2010/0272/2DP/ 2.1.1.1.0/10/APIA/VIAA/088, respectively. The authors would like to thank the European Synchrotron Radiation Facility
(ESRF) for the beamtime at BM29 (project number MA-923), as well as the Hamburger Synchrotronstrahlungslabor (HASYLAB) for the beamtime at beamline A1 (project number I-20090034 EC) and the respective beamline personnel for their support, as well as Sebastian Wicklein and Marcin Wojtyniak for assistance during the beamtimes. The authors thank Dr R. Merkle for the possibility to use reference experimental EXAFS data of the powders solid solutions $\mathrm{SrFe}_{0.1} \mathrm{Ti}_{0.9} \mathrm{O}_{3-x}$, and $\mathrm{P}$. Meuffels and C. Makovicka for providing the $\mathrm{LaFeO}_{3}$ powder.

\section{References}

1 R. Merkle and J. Maier, Angew. Chem., Int. Ed., 2008, 47, 3874-3894.

2 B. P. Andreasson, M. Janousch, U. Staub, T. Todorova, B. Delley, G. I. Meijer and E. Pomjakushina, Phys. Rev. B: Condens. Matter, $2009, \mathbf{8 0}, 212103$.

3 J. Blanc and D. Staebler, Phys. Rev. B: Condens. Matter, 1971, 4, 3548.

4 R. Waser, T. Baiatu and K. Hardtl, J. Am. Ceram. Soc., 1990, 73, 1654-1662.

5 T. Baiatu, R. Waser and K. Hardtl, J. Am. Ceram. Soc., 1990, 73, $1663-1673$.

6 A. Beck, J. Bednorz, C. Gerber, C. Rossel and D. Widmer, Appl. Phys. Lett., 2000, 77, 139-141.

7 R. Waser, R. Dittmann, G. Staikov and K. Szot, Adv. Mater., 2009, 21, 2632.

8 M. Janousch, G. I. Meijer, U. Staub, B. Delley, S. F. Karg and B. P. Andreasson, Adv. Mater., 2007, 19, 2232.

9 G. I. Meijer, U. Staub, M. Janousch, S. L. Johnson, B. Delley and T. Neisius, Phys. Rev. B: Condens. Matter, 2005, 72, 155102.

10 T. Leisegang, H. Stoecker, A. A. Levin, T. Weissbach, M. Zschornak, E. Gutmann, K. Rickers, S. Gemming and D. C. Meyer, Phys. Rev. Lett., 2009, 102, 087601.

11 M. Abbate, F. M. F. deGroot, J. C. Fuggle, A. Fujimori, O. Strebel, F. Lopez, M. Domke, G. Kaindl, G. A. Sawatzky, M. Takano, Y. Takeda, H. Eisaki and S. Uchida, Phys. Rev. B: Condens. Matter, 1992, 46, 4511-4519.

12 K. Szot, W. Speier, G. Bihlmayer and R. Waser, Nat. Mater., 2006, 5, 312-320.

13 A. Kuzmin, R. Kalendarev, A. Kursitis and J. Purans, Latv. J. Phys. Tech. Sci., 2006, 2, 66.

14 A. Kuzmin, Physica B (Amsterdam), 1995, 208, 175-176.

15 A. L. Ankudinov, B. Ravel, J. J. Rehr and S. D. Conradson, Phys. Rev. B: Condens. Matter, 1998, 58, 7565-7576.

16 B. A. Bunker and E. A. Stern, Phys. Rev. B: Condens. Matter, 1983, 27, 1017-1027.

17 M. Vracar, A. Kuzmin, R. Merkle, J. Purans, E. A. Kotomin, J. Maier and O. Mathon, Phys. Rev. B: Condens. Matter, 2007, 76, 174107.

18 S. E. Dann, D. B. Currie, M. T. Weller, M. F. Thomas and A. Alrawwas, J. Solid State Chem., 1994, 109, 134-144.

19 O. Bunau and Y. Joly, J. Phys.: Condens. Matter, 2009, 21, 345501.

20 Y. Joly, Phys. Rev. B: Condens. Matter, 2001, 63, 125120.

21 R. Moos, W. Menesklou and K. H. Hardtl, Appl. Phys. A: Mater. Sci. Process., 1995, 61, 389-395.

22 K. A. Mueller, Helv. Phys. Acta, 1958, 31, 173.

23 E. S. Kirkpatrick, R. S. Rubins and K. A. Muller, Phys. Rev., 1964, 135, A86.

24 R. Merkle and J. Maier, Phys. Chem. Chem. Phys., 2003, 5, $2297-2303$.

25 R.-A. Eichel, Phys. Chem. Chem. Phys., 2011, 13, 368-384.

26 V. E. Alexandrov, J. Maier and R. A. Evarestov, Phys. Rev. B: Condens. Matter, 2008, 77, 075111.

27 J. Hodges, S. Short, J. Jorgensen, X. Xiong, B. Dabrowski, S. Mini and C. Kimball, J. Solid State Chem., 2000, 151, 190-209.

28 J. Blasco, B. Aznar, J. Garcia, G. Subias, J. Herrero-Martin and J. Stankiewicz, Phys. Rev. B: Condens. Matter, 2008, 77, 054107. 